\title{
INDOOR TARGET TRACKING USING HIGH DOPPLER RESOLUTION PASSIVE WI-FI RADAR
}

\author{
Qingchao Chen, Bo Tan, Karl Woodbridge, Kevin Chetty \\ Department of Electronic and Electrical Engineering \\ University College London, London, UK
}

\begin{abstract}
This paper describes two Doppler only indoor passive Wi-Fi tracking methods based on high Doppler resolution passive radar. Two filters are investigated in this paper, the extended Kalman filter and the sequential importance resampling (SIR) particle filter. Experimental results for these two tracking filters are presented using results from software defined passive Wi-Fi radar using a standard 802.11 access point as an illuminator. The experimental results show that the SIR particle filter performs well using Wi-Fi signals for indoor tracking with a high degree of accuracy. Proposals for simplifying the SIR particle and application to multiple target tracking are also discussed.
\end{abstract}

Index Terms - Passive Wi-Fi Radar, Indoor Tracking, Doppler, Kalman Filter, Particle Filter

\section{INTRODUCTION}

Passive radar is a class of radar system for detection and tracking of targets using reflected signals from a noncooperative illuminator. A wide range of commercial broadcast and communications signals have been used in a range of passive radar applications. For example, FM airborne radar [1], WiMAX maritime radar [2], passive GSM radar for vehicle detection [3] as well as DAB+ and DVB-T [4]. The current popularity of the passive concept is related strongly to the increasing pressure on spectrum usage making the use of already available transmitters commercially attractive. In addition the continuing roll out of a wide range of freely available transmissions such as wireless networks means that increasing numbers of potential illuminators of opportunity are available [5]. As in the case of active systems passive radar can also be used for tracking purposes. For example, an FM passive system has been used for tracking airborne targets in [6] by using Gauss-Newton filter. Generally, the tracking methods used in conventional passive radar takes advantage of the bistatic range information [7].

In this paper, a passive radar based on 802.11 (Wi-Fi) transmissions is used to gather data for investigation of indoor target tracking. Use of Wi-Fi signals for indoor positioning is a topic of significant recent interest driven by the increasing public customer marketing demand [8], industrial applications [9] and Internet of Things (IOT) scenarios [10]. So far, there have been a variety of approaches for indoor positioning using Wi-Fi signals. The most widely used method is Wi-Fi fingerprinting [11] which leverages the geographical distribution characteristics of receiving signal strength (RSS) from multiple transmitters to estimate the most likely target location. Apart from RSS, measurement of the time of flight $(\mathrm{ToF})$ or round-trip time (RTT) of an 802.11x package will also be an index of distant between two terminals. Examples of localizing with timing measurements are given in the $802.11 \mathrm{v}$ standard [12] using the hyperbolic method. The angle of arrival (AoA) method has also been used for locating transmission sources normally using antenna arrays as described in [13], [14]. In [15], an outdoor vehicle tracking function is demonstrated using a Wi-Fi AP transmitter and a corresponding receiver array in a passive radar set up by using range and angle information. For the above Wi-Fi tracking approaches, the RSS based methods can reach 1 2 meter accuracy with prerestored RSS database. The localization resolution of the timing based approaches depends on the timing accuracy which can be achieved by software or hardware. The AoA solution generally gives better accuracy, up to 0.5 meters but requires the use of specially designed array antennas and complex signal processing algorithms.

In this paper, a Doppler only tracking approach is proposed for indoor target tracking using transmissions from standard commercial Wi-Fi access points. Doppler only location tracking has mostly been reported for passive airborne targets [6] [16]. In the latter paper a recursive GaussNewton filter is applied for airborne tracking using FM signals. For indoor tracking with Wi-Fi signals, the main concern is the range resolution which is determined by the bandwidth of illuminating of the signal. In the case of Wi-Fi signal, the range resolution could be of the order of 10 to 15 meters which is too coarse for most indoor scenarios. A high Doppler resolution passive Wi-Fi radar using multiple receivers is introduced here for improving this target localization. By fusing the high resolution velocity data from different receivers, high accuracy target detection and tracking can be achieved. There are many important applications for this technique including security, healthcare and retail tracking 


\section{DOPPLER TRACKING USING PASSIVE WIFI RADAR}

2.1. High Doppler Resolution Passive Wi-Fi Radar Most passive radar systems are deployed in a bistatic geometry as shown in Fig. 1. The Doppler in a bistatic system is generated as the target moves between the range ellipse contours.

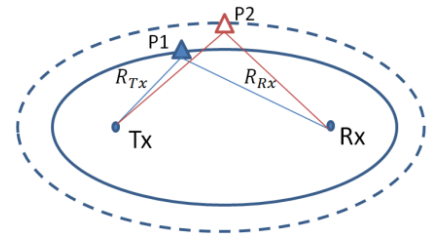

Figure. 1 Bistatic Doppler Generation

As shown in Fig. 1, when the target moves from P1 to P2, the Doppler will be generated as shown in equation (1):

$$
f_{d}=\frac{1}{\lambda} \frac{d}{d t}\left(R_{T x}+R_{R x}\right)
$$

As described in [17], [18], the authors have developed a real-time high Doppler resolution passive Wi-Fi system for indoor target detection. The main components of the system are reference and surveillance antennas ADC modules for each channel and a computing unit for signal processing. In this study we have extended the hardware to use multiple transmit receive pairs and developed and tested algorithms suitable for indoor target tracking.

The system hardware is built around an adaptable software defined radio design. In the computing unit, a down sampling pipelined processing flow is used. This includes normalized least-mean squares (NLMS) DSI filter, batch processing, cell averaging and constant false alarm rate (CFAR) processing. After cross ambiguity function $(\mathrm{CAF})$ processing a range-Doppler matrix is produced. The strongest pixel on this matrix after CFAR application can be extracted and identified as a target. A normalized CAF is used in this work and a target search is perfomed on each sequential CAF matrix generated. The corresponding target Doppler frequency shifts from each CAF are then used for target tracking as described in this paper. The range bins containing the detected target from each CAF are connected together along with a time stamp to form a real time plot of Doppler versus time which can be used for activity classification that is out of the scaope of this paper. The methods in [19] and [20] can be applied to enhance the sharpness of this Doppler record by jointly using the backward and forward detection information.

\subsection{Doppler Only Tracking}

The scenario and the model used in this work are shown in Fig 2. A transmitter, $T X$, (a Wi-Fi Access Point (AP) ) is transmitting a signal with a carrier frequency of $f_{c}$ and several receivers $R X_{i}$ are receiving signals reflected by the moving targets. It is assumed that the location of $T X$ and the $R X_{i}$ are known. The state vector of the target at time $k$ is $x_{k}=\left[x_{k}, y_{k}, v_{x, k}, v_{y, k}\right]^{T}$, where $\left[x_{k}, y_{k}\right]$ is the position, $\left[v_{x, k}, v_{y, k}\right]$ is the velocity and $\mathrm{T}$ is the transpose operation.

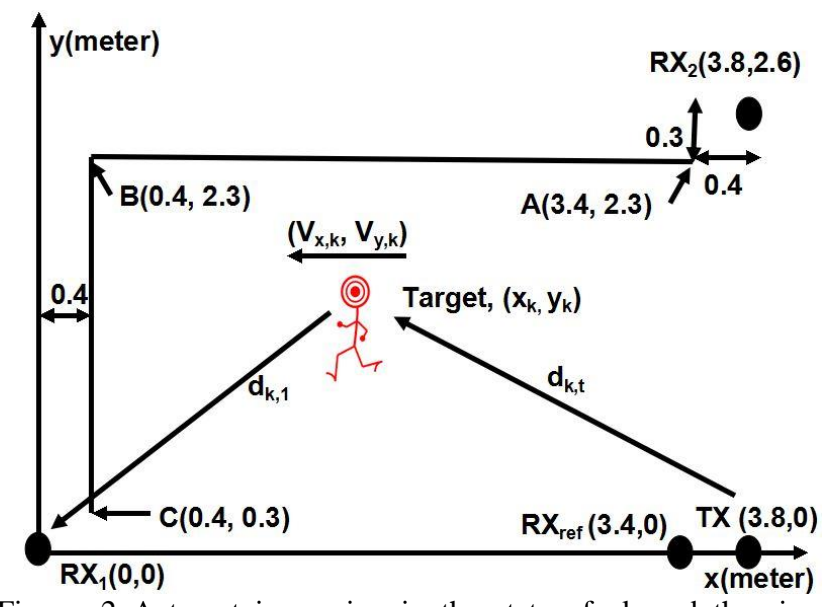

Figure. 2 A target is moving in the state of $\mathrm{xk}$ and the signal transmitted from $T X$ is received by each $R X_{i}, i=1, \ldots, N_{R X} . N_{R X}$ is the total number of receivers

For the state transition function, the constant velocity Gaussian model is used, denoted as follows:

$$
x_{k}=F_{k \mid k-1} * x_{k-1}+V_{k}
$$

where $F_{k \mid k-1}$, the transition matrix can be denoted as:

$$
F_{k \mid k-1}=\left[\begin{array}{cccc}
1 & 0 & \Delta & 0 \\
0 & 1 & 0 & \Delta \\
0 & 0 & 1 & 0 \\
0 & 0 & 0 & 1
\end{array}\right]
$$

The delta is the sampling time interval and $V_{k} \sim N(v ; 0 . Q)$ is the noise of the transition process and the $Q$ is the covariance matrix denoted as:

$$
Q=\sigma_{v}^{2} *\left[\begin{array}{cccc}
\Delta^{4} / 4 & 0 & \Delta^{3} / 2 & 0 \\
0 & \Delta^{4} / 4 & 0 & \Delta^{3} / 2 \\
\Delta^{3} / 2 & 0 & \Delta^{2} & 0 \\
0 & \Delta^{3} / 2 & 0 & \Delta^{2}
\end{array}\right]
$$

where $\sigma_{v}^{2}$ is standard deviation of the transition model noise. The observation model to measure the Doppler information is based on the bistatic radar scenario. Given the position of the transmitter and the $i$ th receiver, $i=1, \ldots, N_{R X}$, the Doppler shifts measured at $i$ th receiver at time $k$, located at $\left[x_{i}, y_{i}\right]$, can be calculated as the following:

$$
Z_{k, i}=h_{i}\left(x_{k}\right)+\varepsilon_{k, i}
$$

where

$$
h_{i}\left(X_{k}\right)=\left\{\frac{v_{x, k}\left(x_{k}-x_{T}\right)+v_{y, k}\left(y_{k}-y_{T}\right)}{\lambda d_{k, T}}\right\}+\left\{\frac{v_{x, k}\left(x_{k}-x_{i}\right)+v_{y, k}\left(y_{k}-y_{i}\right)}{\lambda d_{k, i}}\right\}
$$

is the Doppler shift measurement, $\lambda$ is the wavelength of the transmitted signal and $\varepsilon_{k, i} \sim N\left(\varepsilon ; \sigma_{\epsilon}^{2}\right)$. Distances between the target and the transmitter and $i$ th receiver are denoted as:

$d_{k, T}=\sqrt{\left(x_{k}-x_{T}\right)^{2}+\left(y_{k}-y_{T}\right)^{2}}$ and $d_{k, i}=$ $\sqrt{\left(x_{k}-x_{T}\right)^{2}+\left(y_{k}-y_{T}\right)^{2}}$. 


\subsubsection{Tracking with Extended Kalman Filter (EKF)}

As the observation model is non-linear, EKF is implemented in this work. For transforming the non-linear observation model into the linear one, the first order Taylor expansion is used [21] and the Jacobian matrix of $h_{i}\left(x_{k}\right)$ is denoted as:

$H_{k, i}=\left\{\frac{\partial h_{i}\left(X_{k}\right)}{\partial x_{k}}, \frac{\partial h_{i}\left(X_{k}\right)}{\partial y_{k}}, \frac{\partial h_{i}\left(x_{k}\right)}{\partial v_{x, k}}, \frac{\partial h_{i}\left(x_{k}\right)}{\partial v_{y, k}}\right\}$
where $\frac{\partial h_{i}\left(X_{k}\right)}{\partial x_{k}}=\left\{\frac{v_{x, k} d_{k, T}-\left(x_{k}-x_{T}\right) d_{k, T}}{\lambda d_{k, T}^{2}}\right\}+\left\{\frac{v_{x, k} d_{k, i}-\left(x_{k}-x_{i}\right) d_{k, l}}{\lambda d_{k, i}^{2}}\right\}$,
$\frac{\partial h_{i}\left(X_{k}\right)}{\partial y_{k}}=\left\{\frac{v_{y, k} d_{k, T}-\left(y_{k}-y_{T}\right) d_{k, T}}{\lambda d_{k, T}^{2}}\right\}+\left\{\frac{v_{y, k} d_{k, i}-\left(y_{k}-y_{i}\right) d_{k, i}}{\lambda d_{k, i}^{2}}\right\}$,
$\frac{\partial h_{i}\left(x_{k}\right)}{\partial v_{x, k}}=\left\{\frac{\left(x_{k}-x_{T}\right)}{\lambda d_{k, T}}\right\}+\left\{\frac{x_{k}-x_{i}}{\lambda d_{k, i}}\right\}, \frac{\partial h_{i}\left(X_{k}\right)}{\partial v_{y, k}}=\left\{\frac{\left(y_{k}-y_{T}\right)}{\lambda d_{k, T}}\right\}+\left\{\frac{y_{k}-y_{i}}{\lambda d_{k, i}}\right\}$.

The total derivatives of $d_{k, T}$ and $d_{k, i}$ illustrated in the previous equation are $\dot{d}_{k, T}$ and $\dot{d}_{k, l}$ respectively, represented as the following:

$\dot{d_{k, T}}=\frac{v_{x, k}\left(x_{k}-x_{T}\right)+v_{y, k}\left(y_{k}-y_{T}\right)}{d_{k, T}}, \dot{d_{k, l}}=\frac{v_{x, k}\left(x_{k}-x_{i}\right)+v_{y, k}\left(y_{k}-y_{i}\right)}{d_{k, i}}$.

After calculating the $H_{k, i}\left(X_{k}\right)$, the EKF can be implemented as the following pseudo code. Here, the initial state vector of the target is assumed known in this scenario, denoted as $X_{0}=\left[x_{0}, y_{0}, v_{x, 0}, v_{y, 0}\right]^{T}$ and the mean and covariance matrix of the target's state vector X at time $k$ are denoted as $\mu_{k}$ and $\Sigma_{k}$. The pseudo code of the EKF is shown in Fig. 3.

\subsubsection{Tracking with Sequential Importance Resampling} Particle Filter (SIR PF)

For the implementation of a particle filter, the SIR method is adopted. The initial state vector of the target is $X_{0}=$ $\left[x_{0}, y_{0}, v_{x, 0}, v_{y, 0}\right]^{T}$. Here, the state vector is predicted by $M$ particles according to the transition function $F_{k \mid k-1}$ and the update step is aimed to filter out the particles with the higher weights. Finally, the weighted average state of the particles is the estimated state and the particles are resampled to prepare for the next iteration process [22]. The pseudo code of the SIR particle filter is shown in Fig. 4.

\section{TRACKING EXPERIMENT AND RESULTS}

In this section, the indoor passive $\mathrm{Wi}-\mathrm{Fi}$ radar based tracking experiments are described. The equipment, experimental scenario and system parameters are described and tracking results for both EKF and SIR PF are presented.

\subsection{Experimental Setup}

The experiments were conducted in a standard meeting room within the Engineering Building at UCL. The layout of the experiment is illustrated Figure 2. RX1, RX2, $\mathrm{RX}_{\text {ref }}$ and TX are in at relative positions $(0,0),(3.8,2.6),(3.4,0)$ and $(3.8,0)$ respectively. In the experiments the person target walked from point $\mathrm{A}$ at $(3.4,2.3)$ to point $\mathrm{C}$ at $(0.4$, $0.3)$ via point $\mathrm{B}$ at $(0.4,2.3)$ along the red line shown. For the CAF processing, $0.1 \mathrm{~s}$ of data is used and the FFT length is approximately $0.3 \mathrm{~s}$, that is, the delta in state transition function is $0.1 \mathrm{~s}$. $\sigma_{v}$ is chosen as 0.1 and $\sigma_{\varepsilon}$ is chosen as 0.1 ,
0.5 and 1 . The detailed information about the equipment used in this experiment can be found in [17].

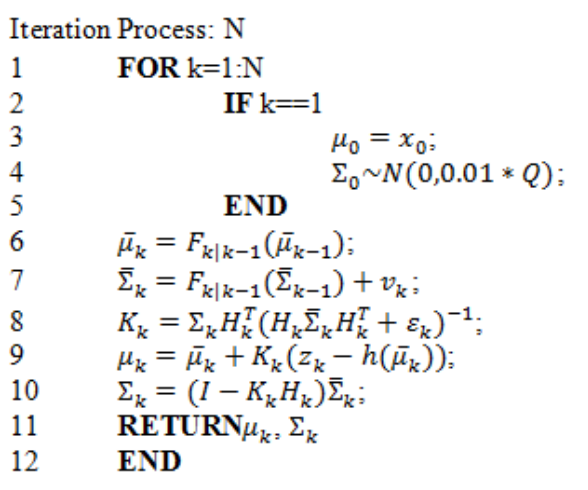

Figure. 3 The pseudo code of EKF tracking

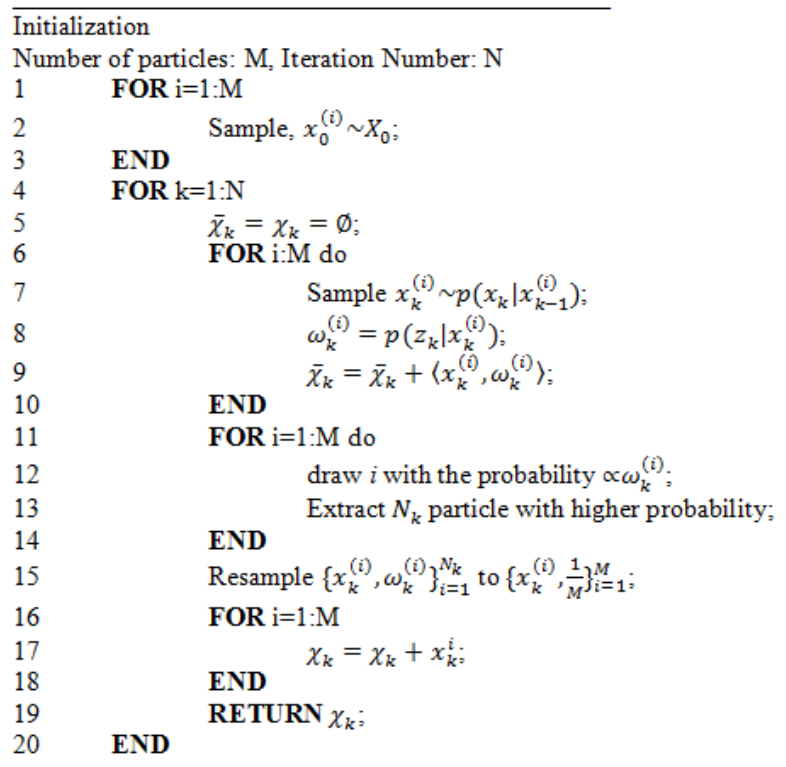

Figure. 4 The pseudo code of SIR PF tracking

\subsection{Tracking Result with Wi-Fi Signal}

\subsubsection{Extended Kalman Filter (EKF)}

The results using EKF are shown in Figure 6 (a). It can be seen that when the target is walking in a straight line in the $\mathrm{X}$ direction, the estimation works well. However, when the target changes direction, the track begins to be lost. This is because of using a linear Gaussian prediction model with EKF which is a combination of Gaussian processes. This is not very suitable for the changes of velocity vector. So in the process of velocity vector adjustment a large amount of noise is generated, especially in the X-axis. The comparatively large track deviation in the $\mathrm{X}$-axis is because when target changes direction suddenly, the velocity in the $\mathrm{X}$ direction is too large to change in one update process while the smaller $\mathrm{Y}$ velocity change can be accommodated. 


\subsubsection{SIR Particle Filter}

The SIR particle filter results are shown in Figures 6 (b), (c) and (d) using 20, 100 and 500 particles. Three tracking results with the same data as the EKF tracking in the previous section are presented. When the target is walking in a straight line from $\mathrm{A}$ to $\mathrm{B}$, the estimation works well and seems as good as the EKF. However, In this case the use of the SIR PF eliminates the need for an assumed probability density function for the Gaussian based prediction and observation process. This means when the target changes direction there is much less track deviation than seen with EKF. As a result, the SIR PF method shows better performance than EKF. As shown in Fig.6 (b), (c) the performance of SIR PF is very dependent upon the number of particles used. It can be seen in Table 1 that using the same parameters, the more particles used in estimation the less the Mean Square Error (MSE). In Table. 1 shows, a comparison between the SIR PF and the EKF results based on the Mean Square Error (MSE). It is concluded that with more measurement noise, the MSE of the filtered results, whether of EKF or PF, is much larger. In addition, with the same covariance, the EKF has a much larger MSE than the SIR PF.

\begin{tabular}{|ll|l|l|l|l|}
\hline & EKF & $\begin{array}{l}\text { SIR PF } \\
(\mathrm{N}=20)\end{array}$ & $\begin{array}{l}\text { SIR PF } \\
(\mathrm{N}=100)\end{array}$ & $\begin{array}{l}\text { SIR PF } \\
(\mathrm{N}=500)\end{array}$ \\
\hline$\sigma_{\mathrm{v}=0.1} \quad \varepsilon=0.1$ & 0.2567 & 0.0889 & 0.571 & 0.0502 \\
\hline$\sigma_{\mathrm{v}=0.1} \quad \varepsilon=0.5$ & 0.2626 & 0.0939 & 0.0799 & 0.0743 \\
\hline$\sigma_{\mathrm{v}=0.1} \quad \varepsilon=1$ & 0.2660 & 0.1031 & 0.0855 & 0.0815 \\
\hline
\end{tabular}

Table. 1: MSE of the results between the EKF and SIR PF, with different measurement noise.
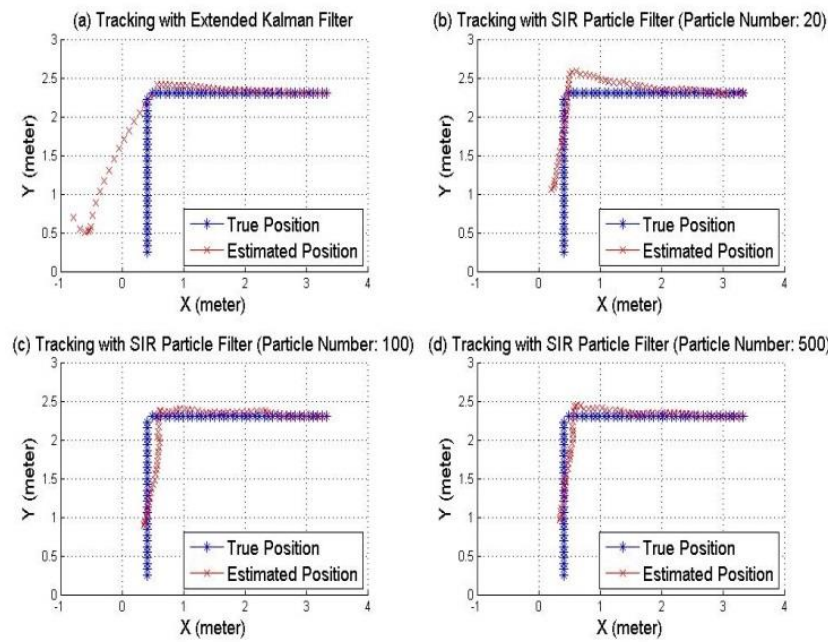

Figure. 6.: (a)Tracking Result Using EKF (b) SIR PF, Particle Number $=20$ (c) SIR PF, Particle Number $=100$ (d) SIR PF, Particle Number $=500$

\subsection{DISCUSSION ON FUTURE WORK}

\subsubsection{Simplification of Particle Filtering}

As can be seen from the above experimental results, the SIR particle filter outperformed the extended Kalman filter in this scenario. This is due to the particle filter avoiding some of the requirements of the Gaussian prediction and measuring processes. The particle filtering mechanism is however constrained by the heavy computing load due to the large number of particles that are required for high performance indoor tracking. As many tracking application will require real-time data, simplifying the SIR particle filtering method should be considered. To achieve this goal, adjusting the resampling mechanism is being considered. In contrast to current resampling methods, the new method uses the rejection mechanism to reduce the particles in each iteration. To keep the performance constant, additional resampling particles are introduced, only when a significant large deviationis observed. For example, when the Doppler measurement changes between samples exceeds a certain threshold the system adds new samples.

\subsubsection{Multiple Indoor Targets}

Multiple target tracking is required in many practical scenarios. Range resolution in long range outdoor scenarios can be quite coarse but needs to be very fine on indoor tracking situations. As previously mentioned, due to the bandwidth of the $\mathrm{Wi}-\mathrm{Fi}$ signal range resolution is not adequate to identify and localize single targets accurately. Therefore to detect and track multiple targets, two approaches could be investigated, secondary elimination of weaker non-target Doppler and the use of the probability hypothesis density (PHD) filter for multiple targets tracking. The secondary elimination is a post processing method which complements the pre NLMS DSI filtering and helps to discover the Doppler generated by the weaker targets which may mask the wanted target. The proposed secondary elimination method can be the scaled frequency shifted CLEAN algorithm [23]. Based on multiple Doppler detections, the PHD is proposed for tracking different targets by selecting from a set of target traces hypothesis.

\section{CONCLUSION}

In this paper we have reported on experimental indoor tracking results using a $\mathrm{Wi}-\mathrm{Fi}$ based passive radar. A Doppler only method has been applied for an indoor scenario to achieve high accuracy tracking in the absence of accurate range information. Doppler only tracking has been demonstrated using both Kalman and Particle filters with data from a software define radio (SDR) passive radar system using genuine $\mathrm{Wi}-\mathrm{Fi}$ transmissions. The SIR particle filter has been shown to outperform the EKF filter when tracking targets with abrupt velocity vector changes. Other new signal processing methods for improving tracking performance for rapidly changing target directions and multiple targets have been suggested. New algorithms for reduction of the signal processing overhead when using particle filtering are being investigated as well as PHD filtering for multiple target tracking. These results represent one of the first detailed studies of indoor target tracking using real passive wireless radar data. 


\section{REFERENCES}

[1] J. Brown, K. Woodbridge, A. Stove, S. Watts, , "VHF airborne passive bistatic radar ground clutter investigation," IET International Conference on Radar Systems (Radar 2012), vol., no., pp.1,5, 22-25 Oct. 2012

[2] K. Chetty, K. Woodbridge, H. Guo, G.E. Smith, "Passive bistatic WiMAX radar for marine surveillance," 2010 IEEE Radar Conference, vol., no., pp.188,193, 10-14 May 2010

[3] P. Samczynski, K. Kulpa, M. Malanowski, P. Krysik, L. Maslikowski, "A concept of GSM-based passive radar for vehicle traffic monitoring," Microwaves, Radar and Remote Sensing Symposium (MRRS), 2011 , vol., no., pp.271,274, 25-27 Aug. 2011

[4] J.E. Palmer, H.A. Harms, S.J. Searle, L.M. Davis, "DVB-T Passive Radar Signal Processing," IEEE Transaction on Signal Processing, vol.61, no.8, pp.2116,2126, April15, 2013

[5] D.W. Bliss, "Cooperative Radar and Communications Signaling:The Estimation and Information Theory Odd Couple", arXiv:1403.1476v1 [cs.IT] 6 Mar 2014

[6] N. Morrison, R.T. Lord, M.R. Inggs, "The Gauss-Newton algorithm in passive aircraft tracking using doppler and bearings," 2007 IET International Conference on Radar Systems, vol., no., pp. $1,5,15-18$ Oct. 2007

[7] Multitarget Tracking Using Multiple Bistatic Range Measurements with Probability Hypothesis Densities

[8] A.H. Lashkari, B. Parhizkar, M.N.A. Ngan, "WIFI-Based Indoor Positioning System," 2010 Second International Conference on Computer and Network Technology (ICCNT), vol., no., pp.76,78, 23-25 April 2010

[9] S. Woo, S. Jeong, E. Mok, L. Xia, C. Choi, M. Pyeon, J. Heo, "Application of WiFi-based indoor positioning system for labor tracking at construction sites: A case study in Guangzhou MTR" Automation in Construction, Pages 3-13, Volume 20, Issue 1, January 2011,

[10] Z. Chen, F. Xia, T. Huang, F. Bu, H.Wang, "A Localization Method for the Internet of Things", Arxiv.org, 2011

[11] I. Bisio, F. Lavagetto, M. Marchese, A. Sciarrone, "Energy efficient WiFi-based fingerprinting for indoor positioning with smartphones," 2013 IEEE Globecom Workshops (GC Wkshps), vol., no., pp.4639,4643, 9-13 Dec. 2013

[12] IEEE 802.11v standard, [Online], Available: http://standards.ieee.org/findstds/standard/802.11v-2011.html

[13] J. Xiong, K. Jamieson, "Array Track: A Fine-Grained Indoor Location System", 10th USENIX Symposium on Networked Systems Design and Implementation, Lombard, US, Apr, 2013

[14] F. Abid, Z. Kabelac, D. Katabi, R.C. Miller "3D Tracking via Body Radio Reflections", 11th USENIX Symposium on Networked Systems Design and Implementation, San Diego, Aug, 2014
[15] P. Falcone, F. Colone, A. Macera, P. Lombardo, "Twodimensional location of moving targets within local areas using WiFi-based multistatic passive radar," IET Radar Sonar \& Navigation, vol.8, no.2, pp.123,131, February 2014

[16] M. Caruso, P. Lombardo, M. Sedehi, "A weighted least squares approach for multi-target Doppler-only localization," 2013 IEEE Radar Conference (RADAR), vol., no., pp.1,6, April 29 2013-May 32013

[17] B. Tan, "Tracking WiFi Signals to Passively See Through Walls Using NI USRP and LabVIEW", National Instrument Case Study http://sine.ni.com/cs/app/doc/p/id/cs-16238 2014.

[18] B. Tan, K. Woodbridge, K. Chetty, "A Real-Time High Resolution Passive WiFi Doppler- Radar and Its Applications" 2014 IEEE International Radar Conference, Lille, Paris, October 2014

[19] K. Yeong-Tae, "Contrast enhancement using brightness preserving bi-histogram equalization," IEEE Transactions on Consumer Electronics, vol.43, no.1, pp.1,8, Feb 1997

[20] Koster, Volker, Andreas Lewandowski, Christian Wietfeld, "A segmentation-based radio tomographic imaging approach for interference reduction in hostile industrial environments." 2012 IEEE Position Location and Navigation Symposium (PLANS), 2012.

[21] G. Welch, G. Bishop. "An introduction to the Kalman filter", University of Nortth Carolina, 2001

[22] J.S. Liu, R. Chen, and T. Logvinenko. "A theoretical framework for sequential importance sampling with resampling." Sequential Monte Carlo methods in practice. p225-246 Springer New York, 2001

[23] K. Chetty, G.E. Smith and K. Woodbridge "Through-TheWall Sensing of Personnel using Passive Bistatic WiFi Radar at Standoff Distances" IEEE Transactions on Geoscience and Remote Sensing. Vol. 99, pp 1-10, 2011.

[24] IEEE 802.11ad standard, [Online], Available: http://standards.ieee.org/findstds/standard/802.11ad-2012.html 